\section{Investigation and management of patients with pleuritic chest pain presenting to the accident and emergency department}

\author{
Kevin Jones, A Raghuram
}

(3) confirming a diagnosis of pulmonary infarction in a patient with chronic obstructive pulmonary disease.

\section{Nature of pleuritic chest pain}

The lung itself is insensitive to pain so the pain of respiratory disease must arise from the pleura, tracheobronchial tree, or chest wall. Pleuritic pain can also be mimicked by cardiac, pericardial, and musculoskeletal disease (see table 1). Pleuritic pain is typically sharp, stabbing, and made worse by inspiration and coughing. Inflammation of the upper part of the parietal pleura causes a pain localised to the chest itself. The lower portion, including the outer segment of the diaphragmatic pleura, is innervated by the lower six intercostal nerves which also supply the abdominal wall. Pleural inflammation here can cause pain in the upper abdomen or loin. ${ }^{2}$ The central part of the diaphragmatic pleura is innervated by the phrenic nerve so that pain from here is felt in the neck and the tip of the shoulder. ${ }^{2}$ The diaphragm can also be irritated by upper abdominal disease such as pancreatitis, cholecystitis, and subphrenic abscess.

Accepted 16 March 1998 pain and an abnormal chest radiograph, and

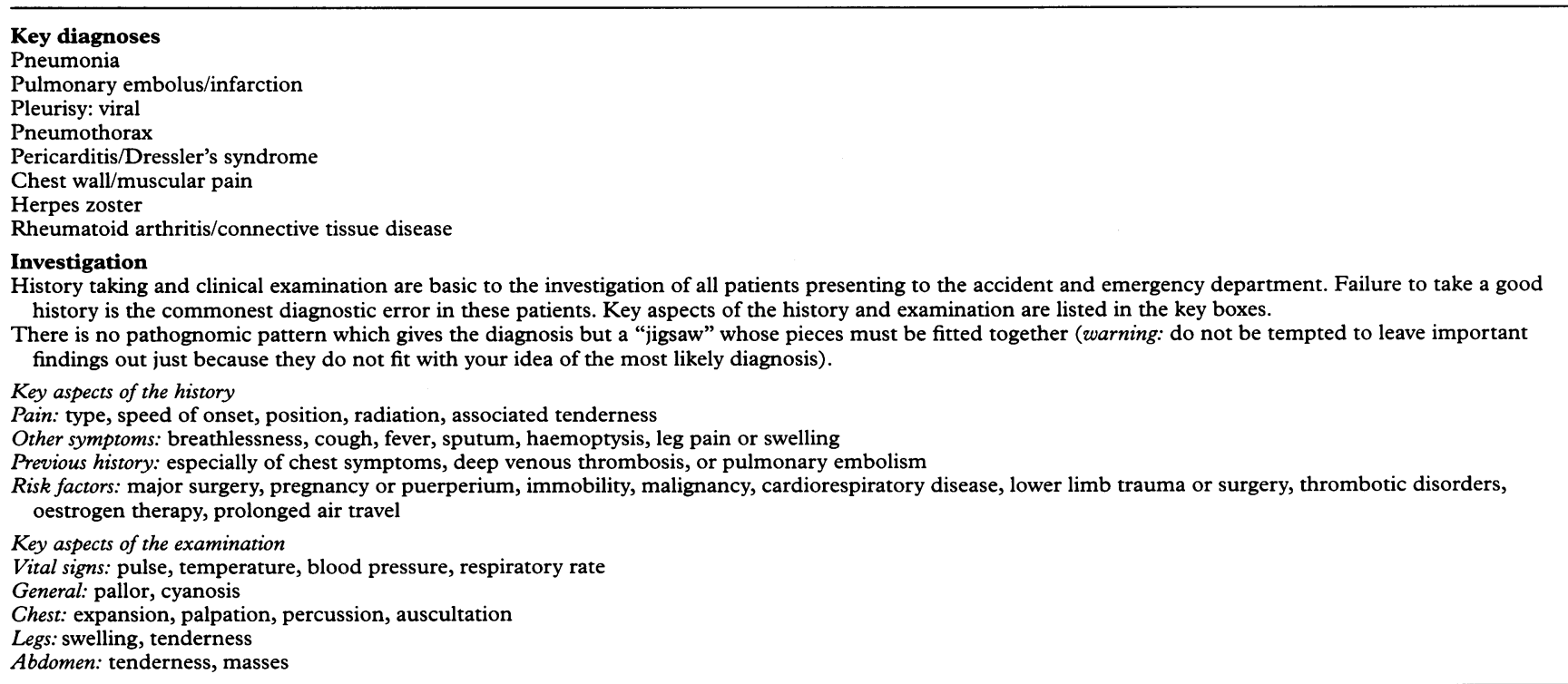


It is important to stress that chest pain is not a universal finding in pulmonary embolus, especially if the embolus is large. However, pleuritic chest pain will occur in $85 \%$ of patients with submassive emboli. ${ }^{3}$ Pulmonary infarction will cause pleuritic chest pain, breathlessness, or both in over $95 \%$ of patients ${ }^{4}$ but the classical triad of pleuritic pain, dyspnoea, and haemoptysis is present in less than a third of patients with documented pulmonary emboli. ${ }^{3-5}$ Ninety per cent of patients with pulmonary embolism will have deep vein thromboses (DVTs) in the leg veins on imaging. ${ }^{6}$ However, clinical signs of DVT may be absent in over $70 \%$ of patients with pulmonary embolism. ${ }^{34}$

\section{Investigations}

A good quality chest radiograph and electrocardiogram (ECG) should be produced in every patient with pleuritic chest pain. Some (especially if breathless with a normal chest radiograph) will also require arterial blood gas analysis to aid with diagnosis. It often helps to go back over the history and examination after the results are available.

THE ECG

The ECG may show classical changes of myocardial infarction or pericarditis, in which case the diagnosis is made and specific treatment can be instituted. The commonest ECG abnormality in pulmonary embolism is simply sinus tachycardia. Atrial fibrillation may also occur. The "classical changes" of pulmonary embolus-"S1,Q3,T3"-are due to right heart strain. They may be seen with large emboli but will be absent in the vast majority. ${ }^{4}$

ARTERIAL BLOOD GASES

Arterial hypoxaemia in a breathless patient without pre-existing lung disease and with a normal chest radiograph is suggestive of pulmonary embolus but is not sensitive. Normal blood gases do not exclude the presence of a small pulmonary embolus. ${ }^{4}$ Other conditions like viral pleurisy can cause similar levels of hypoxia to pulmonary infarction. ${ }^{7}$ Pulse oximetry measures oxygen saturation of haemoglobin. Saturation may remain normal until arterial oxygen pressure has fallen to $8.0 \mathrm{kPa}$. Thus pulse oximetry is a very insensitive measure of hypoxia and is limited in its diagnostic usefulness.

\section{PLEURITIC CHEST PAIN WITH A NORMAL CHEST} RADIOGRAPH

If the chest radiograph is normal, the major differential diagnoses are pericarditis, chest wall pain, viral pleurisy, and pulmonary embolus. The diagnosis is then based on a meticulous history and examination (see above).

(1) Pericardial pain often has a pleuritic component because of associated pleural irritation. It is sometimes brought on by swallowing because of the proximity of the oesophagus to the back of the heart. The pain is worse and more left sided in the supine posture and relieved by leaning forward. There may be an associated pericardial rub which can occur during systole, diastole, or both.

(2) Chest wall pain arising from the intercostal nerves, muscles, or ribs often comes on suddenly after local trauma or violent coughing. Although it hurts to breathe, the patient should not feel breathless when still. The pain may be exacerbated by posture, twisting movements of the thorax, or movements of the upper limbs as well as by inspiration or coughing. There is often a localised area of tenderness on the chest wall and pressure over this should reproduce the pain. However, chest wall tenderness does not completely exclude pulmonary embolism as it can be present in $6 \%$ of such patients. ${ }^{4}$ Bornholm disease is usually caused by the coxsackie $B$ virus. It starts as an upper respiratory tract infection and can cause intercostal myalgia, pleurisy with rub, pericarditis, and chest wall tenderness. ${ }^{8}$ Tietze's syndrome or costochondritis causes painful swelling of the upper costal cartilages. ${ }^{8}$

(3) Viral pleurisy is associated with flu-like symptoms and can cause a pleural rub. It is important to ask all patients about previous prodromal symptoms and to examine for fever and pleural rubs. A rub is thought to be caused by friction between the two layers of inflamed pleura. It is not always accompanied by pain. The sound is classically described as a creak and tends to occur at the same point in each respiratory cycle, often in both inspiration and expiration. It is not removed by coughing and is the one abnormal breath sound that can also be palpable. Its presence means there is pleural inflammation but it does not distinguish the cause. ${ }^{2}$ Fever and leucocytosis can be a feature of both infection and embolism. ${ }^{4}$

The chest radiograph is commonly normal with pulmonary embolism. Its major use is to exclude other pathologies and aid in the interpretation of isotope lung scans. Pulmonary embolism is a difficult condition to diagnose yet failure to do so puts a patient's life at risk. Our advice is that unless there is another obvious explanation for pleuritic chest pain and breathlessness in a patient with a normal chest radiograph, they should be admitted to hospital, started on intravenous anticoagulation with heparin, and then sent for an isotope ventilation/perfusion $(\mathrm{V} / \mathrm{Q})$ scan. This is particularly important if there are any risk factors for pulmonary thromboembolism.

PLEURITIC CHEST PAIN WITH AN ABNORMAL CHEST RADIOGRAPH

The classical features of a pulmonary embolus on chest radiograph include wedge shaped peripheral densities and segmental or lobar diminution in pulmonary vasculature. These are rarely seen. Many of the abnormalities are non-specific, the commonest being atelectasis or pulmonary parenchymal shadows. ${ }^{4}$ Other features include cardiomegaly, pleural effusions, and elevation of the diaphragm. Small pleural effusions occur in $48-60 \%$ of patients with pulmonary infarction and seem to be more common with pulmonary emboli than with other causes of pleurisy. ${ }^{4}$ 
Patients with pleuritic chest pain and an abnormal radiograph often require admission. Patients with a definite diagnosis of pneumonia may be treated according to published guidelines. ${ }^{9}$ It may be very difficult to differentiate between infarction and infection on the basis of the radiograph and if there are features in the history or examination that might suggest pulmonary embolism then these patients will require referral for admission, intravenous anticoagulation, and further investigation. As will be discussed later, if pulmonary infarction causes radiographic abnormalities, it alters the way V/Q scans are interpreted.

\section{PLEURITIC CHEST PAIN AND CHRONIC LUNG} DISEASE

Patients with chronic obstructive pulmonary disease and pleuritic chest pain constitute an extremely difficult diagnostic problem. These patients are at increased risk of pulmonary emboli due to relative immobility, hypoxia, polycythaemia, and right heart failure. However, they also have an increased incidence of infective pleurisy and chest wall pains. Clinical pointers are even more unreliable. For example, chest radiograph, ECG, and arterial blood gases are already likely to be abnormal. In addition, V/Q scanning is unlikely to give a specific answer. The scan will show widespread abnormalities of ventilation and perfusion matching due to the underlying lung disease. It is probably best to refer these patients to the respiratory consultant who is responsible for their long term care.

\section{PULMONARY ANGIOGRAPHY}

Pulmonary angiography is the gold standard for the diagnosis of pulmonary emboli. It is safe, accurate and reproducible, with close agreement in the interpretation of the results between independent observers. However, it is expensive and invasive so that only one third of acute hospitals in Britain supply the service and in these, pulmonary angiography is performed on average only four times a year for diagnosing acute pulmonary emboli. ${ }^{10}$

V/Q LUNG SCANNING

Isotope $\mathrm{V} / \mathrm{Q}$ lung scans are the most commonly used investigation in patients suspected of having pulmonary emboli. Scans are taken of the lungs after the patient breathes a radioactive gas to check for ventilation defects and then again after an intravenous injection of a radioactive marker to check uniformity of perfusion. Evaluation of the scans notes the presence and size (segmental and subsegmental) of perfusion'defects, whether these are matched by defects in ventilation and whether they correlate with abnormalities on the plain chest radiograph. The report is expressed as normal scan, low probability scan, intermediate probability scan or high probability scan. ${ }^{11}$ A normal scan practically rules out the possibility of an acute pulmonary embolus and a high probability scan means the patient should be anticoagulated. Any other scan result is non-diagnostic. Many clinicians believe that reduced perfusion with normal ventilation is the only pattern seen with pulmonary emboli but this is not the case. Pulmonary infarcts can give matched defects. ${ }^{11}$ Therefore, a nondiagnostic scan must be put into the context of the clinical situation. If the clinical suspicion of pulmonary embolus is high then any abnormal scan may justify a diagnosis of pulmonary embolism and appropriate anticoagulant treatment.

If pulmonary infarction causes radiographic evidence of pulmonary infiltrates, it makes $V / Q$ scanning difficult to interpret. The V/Q scan is likely to show non-diagnostic changes in the area of the chest radiographic shadowing which will make differentiation between infarct and other pathologies (for example pneumonia) impossible. If there are additional $V / Q$ defects in areas which appear normal on the chest radiograph, then this is supportive of a diagnosis of pulmonary emboli. " If the $V / Q$ scan is non-diagnostic (which occurs in about $70 \%$ of cases $^{11}$ ) imaging of the deep venous system of the legs has been suggested as a way of further determining which patients require anticoagulation, which require further investigation, and which can be safely observed without specific treatment. ${ }^{1213}$

\section{DETECTION OF DVT}

(This subject is covered in another article in the series.)

DVT in the calf veins is not a risk factor for pulmonary emboli. Venous thrombi in popliteal veins or above are highly likely to cause clinically significant pulmonary emboli. Many DVTs (including those in more proximal veins) occur without symptoms or signs. ${ }^{14}$ Ascending venography is the gold standard for detecting DVTs but this is invasive, expensive, and painful so most hospitals rely on Doppler ultrasound or impedance plethysmography. Neither test is able to detect calf vein thromboses, but will detect the more significant proximal vein thrombi. Both tests have sensitivities and specificities of over $90 \%$ in patients with symptoms suggestive of DVT.

Venograms show thrombus in the deep venous system in at least $70 \%$ of patients with pulmonary emboli. This has led to the theory that if a patient with presumed pulmonary embolism has no evidence of venous thrombosis, then either the diagnosis is wrong or the thrombus has completely broken off and there is little chance of further emboli. ${ }^{15}$ It is now commonly advocated that any patient with a non-diagnostic V/Q scan should have Doppler ultrasound of the lower limbs. If this shows thrombi, then the diagnosis of pulmonary embolism is likely and the patient should be anticoagulated. Problems arise if no DVT is demonstrated (as occurs in about $40 \%$ of patients with suspected pulmonary embolism ${ }^{15}$ ). In the larger centres, pulmonary angiography is advocated, but as already discussed, this is unlikely to be feasible in most district general hospitals. Although the patient could be transferred to a unit that regularly practices pulmonary angiography, this is highly impracticable due to the large number 
of patients involved. Some centres do not anticoagulate but carry out serial investigations of the deep veins of the legs and proceed to anticoagulation if a proximal DVT arises. $^{15}$

Often, the decision on whether or not to continue anticoagulation after a nondiagnostic scan and no evidence of DVT comes down to the clinical suspicion of pulmonary embolism and the presence of associated risk factors. It is not uncommon to treat patients for both pneumonia and pulmonary embolism with intravenous heparin and antibiotics in the short term and warfarin for longer. This is probably a safer course of action than not to anticoagulate if any doubt remains. We believe the consequences of a further large pulmonary embolus outweigh the risks of side effects from warfarin. This is especially so when warfarin does not have to be taken long term. The British Thoracic Society recommends that in the presence of a known risk factor, a patient diagnosed as having a pulmonary embolism need be anticoagulated for only four weeks (provided the risk factor has been removed ). If there is no identifiable risk factor, then warfarin should be given for three months. The incidence of major haemorrhagic complications in these two groups of patients is very low at less than $2 \% .^{16}$

Patient with pleuritic chest pain

Other diagnosis confirmed

Pulmonary embolism (PE) suspected

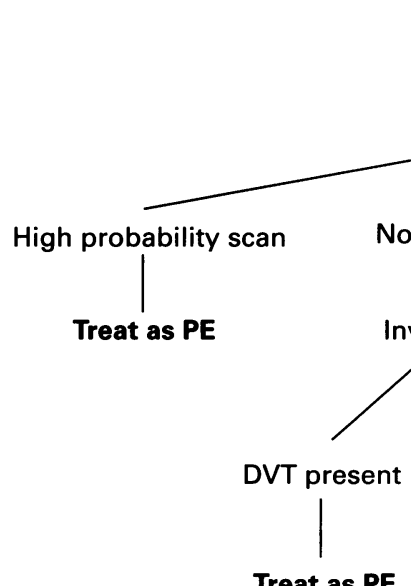

Heparinise

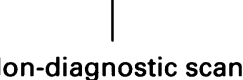

nvestigate for DVT

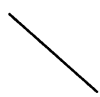

Do not treat as PE

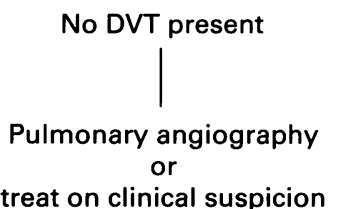

treat on clinical suspicion

Figure 1 Algorithm for investigation and management of pleuritic chest pain. $C X R=$ chest radiograph; $D V T=$ deep vein thrombosis; $E C G=$ electrocardiogram, $V / Q=$ ventilation/perfusion.

D-DIMER AND SPIRAL COMPUTED TOMOGRAPHY $\mathrm{D}$-dimer is a fragment produced by the action of the fibrinolytic system. Its levels are raised in pulmonary embolism. It seems to be a highly sensitive test but unfortunately is very non-specific. If low levels of D-dimer were found in suspected pulmonary embolism with a non-diagnostic V/Q scan and negative investigations for DVT, this might give further reassurance that anticoagulation was unnecessary. ${ }^{17}$ Probably the best advance for the future will be the introduction of a non-invasive imaging technique to replace pulmonary angiography. Spiral volumetric computed tomographic scanning may provide such a technique which could be readily used in every district general hospital. At present, however, doubts remain about its ability to detect small peripheral pulmonary emboli. ${ }^{18}$

\section{British Thoracic Society guidelines}

The British Thoracic Society has recently produced guidelines for the investigation and management of patients with suspected pulmonary embolism. ${ }^{19}$ These give a full review of the literature with graded recommendations and levels of evidence. Their principal aim is to recommend a diagnostic and management strategy which could be used by junior medical staff. We strongly advocate that our colleagues in $A \& E$ medicine become familiar with these guidelines.

\section{Summary}

The assessment of a patient with pleuritic chest pain calls for a high degree of clinical acumen and a high degree of suspicion that the diagnosis might be pulmonary embolism. This area is one of the most difficult in A\&E medicine (and indeed chest medicine). One error is to "think the best" when considering the diagnosis in such patients but experience soon teaches to "think PE" and diagnose less serious conditions only when pulmonary embolism has been excluded. A key consideration is the presence of risk factors. Because the diagnosis is difficult, there should be no hesitation in requesting a senior opinion or referring to the inpatient medical team. We have produced an algorithm (fig 1) for the investigation and management of pleuritic chest pain as discussed in this article.

Three questions relating to this article are:

(1) Can pulmonary embolism be the diagnosis in a patient with pleuritic chest pain but a normal chest radiograph, ECG, and arterial blood gases?

(2) What is the chest radiograph abnormality which is most likely to alert you to the possibility of pulmonary embolism?

(3) What percentage of patients with a low clinical suspicion of pulmonary embolism but a high probability V/Q scan will have pulmonary embolism demonstrated on pulmonary angiography? (see Fennerty ${ }^{13}$ )

The three key references are The PIOPED Investigators, ${ }^{11}$ Dalen, ${ }^{12}$ and Fennerty. ${ }^{13}$ 
1 Hull RD, Raskob GE, Carter CJ, et al. Pulmonary embolism in outpatients with pleuritic chest pain. Arch Intern Med 1988;148:838-44.

2 Ogilvie CM. Symptoms and signs in respiratory disease. In Brewis RAL, Corrin B, Geddes DM, Gibson GJ, eds. Respiratory medicine. 2nd Ed. London: WB Saunders, 1995: 245-57.

3 Bell WR, Simon TL, DeMets DL. The clinical features of submassive and massive pulmonary emboli. $\mathrm{Am} \mathrm{f}$ Med 1977;62:355-60

4 Stein PD, Terrin ML, Hales CA, et al. Clinical, laboratory, roentgenographic and electrocardiographic findings in patients with acute pulmonary embolism and no prepatients with acute pulmonary embolism and no pre598-603.

5 Wenger NK, Stein PD, Willis PW. Massive acute pulmonary embolism. The deceivingly nonspecific manifestations. भAMA 1972;220:843-4.

6 Hull R, Hirsh J, Carter CJ, et al. Pulmonary angiography, ventilation lung scanning and venography for clinically suspected pulmonary embolism with abnormal perfusion scans. Ann Intern Med 1983;98:891-9.

7 Branch WT, McNeil BJ. Analysis of the differential diagnosis and assessment of pleuritic chest pain in young adults. Am $\mathcal{F}$ Med 1983;75:671-9.

8 Macfarlane JT. Acute respiratory infections in adults. In: Brewis RAL, Corrin B, Geddes DM, Gibson GJ, eds. Respiratory medicine. 2nd Ed. London: WB Saunders, 1995: 705-46.

9 British Thoracic Society. Guidelines for the management of community acquired pneumonia in adults admitted to hospital. Brf Hosp Med 1993;49:346-50.
10 Cooper TJ, Hayward WJ, Hartog M. Survey on the use of pulmonary scintigraphy and angiography for suspected pulmonary thromboembolism in the UK. Clin Radiol 1991;43:243-5.

11 The PIOPED Investigators. Value of the ventilation/ perfusion scan in acute pulmonary embolism. $\mathcal{F} A M A$ 1991;263:2753-9.

12 Dalen JE. When can treatment be withheld in patients with suspected pulmonary embolism? Arch Intern Med 1993; 153:1415-8.

13 Fennerty $\mathrm{T}$. The diagnosis of pulmonary embolism. BMF 1997;314:425-9.

14 Moser KM. State of the art. Venous thromboembolism. $\mathrm{Am}$ Rev Respir Dis 1990;141:235-49.

15 Hull RD, Raskob GE, Coates G, et al. A new noninvasive management strategy for patients with suspected pulmonary embolism. Arch Intern Med 1989;149:2549-55.

16 Research Committee of the British Thoracic Society. Optimum duration of anticoagulation for deep venous thrombosis and pulmonary embolism. Lancet 1992;340:873-6.

17 Mountain D, Brown AFT. A review of the usefulness of Mountain D, Brown AFT. A review of the usefulness of
D-dimer in the diagnosis of pulmonary embolism. D-dimer in the diagnosis of
Emergency Medicine 1996;8:253-9.

18 van Rossum AB, Treurniet FEE, Kieft GJ, et al. Role of spiral volumetric computed tomographic scanning in the assessment of patients with clinical suspicion of pulmonary embolism and an abnormal ventilation/perfusion lung scan. Thorax 1996;51:23-8.

19 British Thoracic Society, Standards of Care Committee. Suspected acute pulmonary embolism: a practical approach. Thorax 1997;52 (suppl 4): S1-24. 